\title{
Niveles de glucemia en recién nacidos a término, adecuados para la edad gestacional, alimentados con leche materna exclusiva y no exclusiva
}

\author{
Custodio Quispe ${ }^{1,2}$, Ricardo Terukina ${ }^{3}$
}

Resumen

Palabras clave

\begin{abstract}
Introducción: Los recién nacidos ( $R N$ ) alimentados con leche materna exclusiva (LME) pueden presentar niveles bajos de glucemia en las primeras horas de su vida y su monitoreo es muy importante. Las hipoglucemias severas están asociadas con secuelas neurológicas. Objetivos: Determinar la influencia de la lactancia materna exclusiva y no exclusiva sobre los niveles de glucemia en el neonato, durante las primeras 24 horas de vida. Diseño: Estudio de tipo cuasiexperimental. Lugar: Servicios de Atención Inmediata y Puericultura, Instituto Nacional Materno Perinatal. Participantes: Recién nacidos. Intervenciones: Se estudió a 90 RN distribuidos en 3 grupos, según modalidades de alimentación: a) 30 con LME, b) 30 con leche materna más leche maternizada y c) 30 con leche materna más suero glucosado. Se captó al RN que reunía los criterios de inclusión y previo consentimiento informado de la madre, se tomó la muestra de sangre a la madre y al neonato, para determinar la glucemia al nacimiento, a las 2, 4, 6, 12, 18 y 24 horas. Principales medidas de resultados: Valores de glucemia en el recién nacido. Resultados: Los RN alimentados con LME tuvieron una tendencia a la disminución de los niveles de glucemia más prolongada y un incremento más tardío del mismo, que los alimentados con leche materna más leche maternizada y leche materna más suero glucosado, siendo estas diferencias altamente significativas ( $p<0,0001$ y $p=0,006$ respectivamente). Conclusiones: El nivel de glucemia menor a $40 \mathrm{mg} / \mathrm{dL}$ (hipoglucemia) fue mayor en los RNalimentados con LME (16,7\%), siguiéndole los alimentados con leche materna más leche maternizada (10\%) y ninguno en los alimentados con leche materna más suero glucosado. La pérdida ponderal fue mayor en los neonatos alimentados con LME.
\end{abstract}

Recién nacido; lactancia materna; edad gestacional; hipoglicemia.
Levels of glycemia in at term newborns adequate for gestational age breast-fed exclusively or not

Abstract

Introduction: Newborns exclusively breast-fed(EBF) maypresent low levels of glycemia in the first hours of life and their monitoring is very important. Severe hypoglycemias are associated with neurological sequels. Objectives: To determine the influence of breastfeeding exclusive or not over glycemia levels in the newborn during the first 24 hours of life. Design: Cuasiexperimental kind of study. Setting: Immediate care and child care services, Instituto Nacional Materno Perinatal, Lima, Peru, a teaching hospital.

1 Instituto Nacional Materno Perinatal, Ministerio de Salud. Lima, Perú.

2 Departamento Académico de Pediatría. Facultad de Medicina, UNMSM. Lima, Perú.

3 Departamento Académico de Medicina Preventiva y Salud Pública. Facultad de Medicina, UNMSM. Lima, Perú.
Participants: Breast-fed newborns. Interventions: Ninety newborns were distributed in 3 groups according to the kind of feeding: a) $30 \mathrm{EBF}$; b) 30 breastfeeding plus maternized milk; and,c) 30 breastfeeding plus glucose solution. Newborns admited fulfilled criteria of inclusion and previous consent of the mother a sample of blood was taken from the mother and the newborn to determine glycemia at birth and at 2, 4, 6, 12, 18, and 24 hours. Main outcome measures: Newborn glycemia levels. Results: Newborns EBF have a tendency to a more prolonged decrease in glycemia levels and a farther increase, than those breastfed plus maternized milk and breast milk plus glucose solution; these diferences were significant with a $p<0,0001$ and $p=0,006$ respectively. Conclusions: The levels of glycemia $<40 \mathrm{mg} / \mathrm{dL}$ (hypoglycemia) was higher in those EBF (16,7\%), followed by those breast-fed plus maternized milk (10\%) and none in those breast-fed plus glucose solution. Weight lose was greater in those newborns with EBF.

Key words: Infant, newborn; breast feeding; gestacional age; hypoglycemia. 


\section{INTRODUCCIÓN}

La determinación de la glucosa sanguínea es parte inherente de los cuidados básicos neonatales en los establecimientos de salud. Sin embargo, los niveles sanguíneos de glucosa normal no están clínicamente precisados y, por consiguiente, su interpretación continúa siendo un reto para el médico neonatólogo $\left({ }^{1}\right)$. La glucosa es un substrato muy importante en el metabolismo de las células, especialmente de las neuronas. Las hipoglucemias neonatales severas y prolongadas están asociadas a largo plazo con secuelas neurológicas en el desarrollo del niño $(2,3)$. Actualmente, se define la hipoglucemia neonatal como la concentración sanguínea de glucosa menor a $40 \mathrm{mg} / \mathrm{dL}(2,2 \mathrm{mOsm} /$ L), cualquiera que sea su peso y edad gestacional al nacer $\left(^{4-6}\right)$. El grupo de trabajo sobre lactancia materna de la Academia Americana de Pediatría considera que la leche materna es netamente superior para la alimentación del lactante y tiene carácter específico para la especie $\left(^{7}\right)$. El niño alimentado con leche materna exclusiva (LME) es el modelo de referencia o la norma frente a la cual deben compararse todos los demás métodos de alimentación, en lo que respecta a diversos parámetros, tales como crecimiento, estado de salud, desarrollo, nutrición, nivel inmunológico y otros, a corto y a largo plazo $\left(^{5,7}\right)$. La lactancia materna debe comenzar lo más pronto posible después del nacimiento; en general dentro de la primera hora, y luego continuar a libre demanda. El hecho de que la madre comparta la cama con el recién nacido (RN) en el Servicio de Alojamiento Conjunto favorece la lactancia materna exclusiva, observándose un efecto visible sobre el incremento ponderal 'después de la bajada de leche' ${ }^{(7,8)}$. En 1991, surgió la Iniciativa del Hospital Amigo del Niño, que fue un intento internacional por parte de la OMS y UNICEF para estimular, proteger y apoyar el amamantamiento en hospitales y centros natales a nivel mundial $\left.{ }^{9}\right)$. En el Perú, el Instituto Nacional Mater- no Perinatal (INMP) fue uno de los primeros hospitales en recibir la Certificación Hospital Amigo de la Madre y el Niño, en agosto de 1994 , por haber puesto en práctica los diez pasos hacia una lactancia natural exitosa. La lactosa constituye 7 a $8 \%$ de los nutrientes de la leche humana y de las fórmulas lácteas 'maternizadas' comerciales, lo que representa 35 a $40 \%$ del total de las calorías $\left({ }^{10}\right)$. La lactasa, enzima encargada de hidrolizar a la lactosa en glucosa y galactosa, aparece durante el tercer mes de la gestación, permanece en niveles bajos hasta el sexto mes, aumenta durante el tercer trimestre y alcanza sus valores máximos en torno al nacimiento. La lactasa tiene una localización más apical en la vellosidad intestinal, lo que explica su mayor susceptibilidad a las lesiones agudas de la mucosa, como ocurre en las gastroenteritis virales $\left({ }^{10,11}\right)$. La glucosa es esencial para la nutrición del cerebro y del sistema nervioso periférico, para las células de la sangre y la médula renal. La glucosa se almacena en forma de glucógeno en el hígado $\mathrm{y}$, en el neonato a término, este glucógeno puede llegar al 5\% $\left({ }^{12,13}\right)$. El transporte activo de la glucosa y de la galactosa se producen en dos etapas: la primera es la acumulación del azúcar dentro del epitelio (enterocito), por acción del cotransportador $\mathrm{Na}^{+}$glucosa-galactosa (SGLT1); la segunda etapa consiste en el transporte de la glucosa hacia la sangre, a través de la membrana basolateral, por medio del uniportador GLUT2 $\left({ }^{11,12,14,15}\right)$. Existen métodos rápidos y sencillos para diagnosticar y tratar la hipoglucemia. A lo largo de la historia, han mejorado los métodos de medición de la glucosa sanguínea, cada vez con técnicas más exactas y precisas, utilizando un pequeño volumen de sangre de apenas $2,5 \mathrm{uL}$ y obteniéndose los resultados rápidamente en un tiempo de 20 segundos $\left({ }^{16}\right)$.

En el Servicio de Puericultura Neonatal del Departamento de Neonatología del INMP, donde están alojados las parejas ma- 
dre-hijo sanos, compartiendo la misma cama con indicación de lactancia materna exclusiva para el neonato, se observó algunos casos de hipoglucemia neonatal, lo que hizo necesario el apoyo con sucedáneos de leche materna o suero glucosado, a fin de mejorar los niveles bajos de glucemia, hecho que transgrede las recomendaciones del grupo de trabajo sobre lactancia materna de la Academia Americana de Pediatría, sobre la alimentación del lactante $\left({ }^{7,17}\right)$. La prevención de la hipoglucemia neonatal comienza con un buen control del embarazo y una cuidadosa monitorización de la glucemia materna durante el parto. La alimentación al pecho mejora también el vínculo emocional entre la madre y el niño $(5,7,18)$.

La situación descrita destaca la importancia y necesidad de realizar el presente estudio, el que se desarrolló durante los meses de agosto 2004 a enero 2005.

Los objetivos del estudio fueron: a) Determinar los niveles de glucemia en los RN alimentados con LME y leche materna no exclusiva, durante las primeras 24 horas de vida; b) Establecer la relación entre las diversas modalidades de alimentación del RN y los niveles de glucemia; c) Determinar la incidencia de hipoglucemia en las tres modalidades de alimentación; y, d) Establecer la relación entre los niveles de glucemia del RN y los niveles de glucemia materna.

\section{MATERIALES Y MÉTODOS}

El presente trabajo de investigación fue de tipo cuasiexperimental. La población de estudio estuvo constituido por los recién nacidos en el INMP y fue de tipo dinámico, debido al poco tiempo de estancia hospitalaria. Los criterios de inclusión fueron: RN de parto eutócico, sanos, a términos y adecuados para la edad gestacional, con contacto precoz de piel a piel con la madre, alimentados con leche materna inme- diatamente después del parto y que compartían la misma cama con su madre, las 24 horas del día. Y los criterios de exclusión fueron: RN con líquido amniótico meconial, de madre con hipertensión, toxemia. La unidad de muestreo y análisis fue el $\mathrm{RN}$, el método de muestreo fue no probabilístico, por conveniencia y disponibilidad en el lugar y el momento del estudio. El estudio quiso establecer el efecto de tres dietas alimenticias en el nivel de glucemia de los RN (uno de ellos considerado como control). El tamaño de la muestra se estimó utilizando las fórmulas estadísticas para la comparación de las medias de tres muestras independientes $\left({ }^{19}\right) \mathrm{y}$, de acuerdo a la misma, el estudio requirió 30 neonatos por cada modalidad de alimentación. Las variables independientes fueron las diversas modalidades de alimentación: 1) alimentación con LME; 2) alimentación con leche materna no exclusiva: a) Leche materna más leche maternizada. b) Leche materna más solución de dextrosa al 5\%. La variable dependiente fue: niveles de glucemia en el RN.

Una vez ocurrido el período expulsivo, el RN que cumplía los criterios de inclusión y exclusión fue captado para el estudio y, previo consentimiento informado de la madre, se tomó la muestra de sangre a la madre y al RN para determinar los valores de glucemia, utilizando tiras reactivas. La modalidad de alimentación del RN se asignó al azar, en forma secuencial: al primero se le asignó al grupo con LME, al segundo con leche materna más leche maternizada de inicio, al tercero con leche materna más el aporte de dextrosa al 5\%, y así sucesivamente se procedió con los demás RN, hasta completar el tamaño de la muestra requerida. Posteriormente, al neonato se le extrajo muestras de sangre para la determinación de los niveles de glucemia, a las 2, 4, 6, 12, 18 y 24 horas de vida. La toma de muestra se realizó en el talón izquierdo de los $\mathrm{RN}$; la primera toma se realizó antes de los $30 \mathrm{mi}-$ 
nutos de vida. La toma de muestra de sangre en la madre fue realizada en el dedo índice de la mano derecha, dentro de los 30 minutos de haber ocurrido el parto.

Cuando se aplica de forma inmediata la muestra de sangre total capilar fresca a la tira reactiva, la glucosa presente en la sangre reacciona con los productos químicos de ésta. Esto produce una pequeña corriente eléctrica y el resultado de la glucemia aparece en la pantalla del glucómetro Optium.- Medisence de Laboratorios Abbot, que tiene un rango de 20 a $500 \mathrm{mg} / \mathrm{dL}$. La magnitud de la corriente depende de la cantidad de glucosa presente en la muestra de sangre. El área de cada tira reactiva (electrodo) contiene glucosa oxidasa (Aspergillus niger $) \geq 0,02$ UI e ingredientes no reactivos $\geq 60,0 \mathrm{ug}$. Es consenso generalizado de que el patrón de oro o referencia de la medición de glucosa sanguínea lo constituye el método de laboratorio de la glucosa hexoquinasa $\left({ }^{16}\right)$.

Para el plan de tabulación y análisis de los datos se utilizó el paquete estadístico SPSS versión 13,0. Las variables cualitativas se describe como frecuencia y porcentajes, y las cuantitativas se presenta como promedio y desviación estándar. Para evaluar la asociación de variables se aplicó la prueba de chi cuadrado, y las mediciones repetidas de glucosa de las diversas modalidades de alimentación fueron analizadas con el ANOVA multivariante para muestras repetidas. Se consideró significativos valores de $p \leq 0,05$. El presente estudio fue aprobado por el Comité de Ética del INMP. Se tomó en cuenta las recomendaciones de la Declaración de Helsinski (revisión del año 2000) y las Normas Éticas Internacionales para las Investigaciones Biomédicas con Sujetos Humanos, elaboradas por el Consejo de Organizaciones Internacionales de las Ciencias Médicas (CIOMS), en colaboración con la OMS, publicadas en $1996\left({ }^{20}\right)$.

\section{RESULTADOS}

En relación con las características maternas y de los RN, se observó que los valores promedios y porcentuales de diversas variables estaban dentro de límites normales. Y al comparar las características maternas y de los $\mathrm{RN}$, según modalidades de alimentación, observamos que las cifras promedio de la mayor parte de las variables fueron similares en los diversos grupos, salvo la hemoglobina materna y el perímetro cefálico del $\mathrm{RN}$, cuyas diferencias fueron significativas $(p=0,028$ y $p=0,019$, respectivamente). Debemos destacar que la glucemia basal de los RN que posteriormente fueron alimentados con leche materna más suero glucosado fue discretamente más elevada que en los que recibieron LME y leche materna más leche maternizada de inicio, aunque estas diferencias no fueron significativas $(p>0,05)$ (Tabla 1). En la distribución de los RN por modalidades de alimentación, según estado civil y grado de instrucción de las madres, predominaron las madres convivientes y las de instrucción secundaria, con $74,4 \%$, no hallándose diferencias significativas entre los tres grupos de estudio.

Al observar la evolución de los niveles de glucemia de los RN en las tres modalidades de alimentación por sexo (Tabla 2), hallamos que los varones tienen una tendencia al descenso hasta las 2 horas de vida y luego se incrementa a partir de las 4 horas; y, en las mujeres, este descenso se prolonga hasta las 4 horas de vida y el incremento ocurre a partir de las 6 horas, tanto en los alimentados con LME y leche materna más leche maternizada de inicio. Sin embargo, en los RN alimentados con leche materna más suero glucosado, esta tendencia al descenso es más corta (hasta las 2 horas de vida) y se incrementa a partir de las 4 horas, tanto en varones como en mujeres. Las diferencias en los descensos e incrementos de los niveles de glucemia en 
Tabla 1. Características maternas y de los recién nacidos según modalidad de alimentación.

\begin{tabular}{|c|c|c|c|c|c|c|c|}
\hline \multirow[t]{3}{*}{ Características } & \multicolumn{6}{|c|}{ Modalidad de alimentación } & \multirow{3}{*}{$\begin{array}{l}\text { Valor } \\
\text { de } p\end{array}$} \\
\hline & \multicolumn{2}{|c|}{$\begin{array}{l}\text { Leche materna } \\
\text { exclusiva }\end{array}$} & \multicolumn{2}{|c|}{$\begin{array}{l}\text { Leche materna más leche } \\
\text { maternizada de inicio }\end{array}$} & \multicolumn{2}{|c|}{$\begin{array}{c}\text { Leche materna } \\
\text { más suero glucosado }\end{array}$} & \\
\hline & Promedio & $\overline{\mathrm{DE}}$ & Promedio & $\mathrm{DE}$ & Promedio & $\overline{\mathrm{DE}}$ & \\
\hline \multicolumn{8}{|l|}{ Maternas } \\
\hline Edad, en años & 24,8 & 5,7 & 25,5 & 6,1 & 24,0 & 6,0 & 0,633 \\
\hline Peso, en kg & 55,3 & 8,5 & 55,4 & 8,2 & 56,8 & 7,0 & 0,512 \\
\hline Talla, en cm & 154,8 & 5,5 & 154,5 & 4,7 & 153,3 & 6,7 & 0,611 \\
\hline $\operatorname{IMC}\left(\mathrm{kg} / \mathrm{m}^{2}\right)$ & 22,6 & 4,2 & 234,4 & 3,1 & 23,9 & 2,8 & 0,402 \\
\hline Glucemia (mg/dL) & 105,1 & 16,7 & 112,9 & 22,4 & 110,6 & 28,3 & 0,407 \\
\hline Hemoglobina, en g/L & 11,3 & 0,8 & 10,8 & 1,4 & 11,7 & 1,0 & 0,028 \\
\hline \multicolumn{8}{|l|}{ Recién nacidos } \\
\hline Edad gestacional (semanas) & 39,4 & 0,8 & 39,6 & 0,9 & 39,7 & 0,7 & 0,181 \\
\hline Peso al nacer, en gramos & 3319,0 & 267,2 & 3467,3 & 302,3 & 3325,3 & 328,9 & 0,103 \\
\hline Talla, en cm & 50,2 & 1,5 & 50,6 & 1,3 & 49,9 & 1,5 & 0,145 \\
\hline Índice ponderal (kg/m³) & 26,0 & 0,2 & 27,7 & 0,2 & 27,0 & 0,2 & 0,642 \\
\hline Perímetro cefálico, en cm & 34,4 & 0,8 & 34,9 & 1,0 & 34,2 & 1,0 & 0,019 \\
\hline Temperatura, en ${ }^{\circ} \mathrm{C}$ & 36,8 & 0,4 & 36,9 & 0,3 & 36,9 & 0,4 & 0,653 \\
\hline Glucemia al nacer (mg/dL) & 65,0 & 14,4 & 65,5 & 14,9 & 69,9 & 15,3 & 0,371 \\
\hline Glucemia RN/materna (\%) & 63,1 & 15,6 & 58,8 & 12,3 & 65,3 & 14,6 & 0,210 \\
\hline
\end{tabular}

$n=90 \quad D E=$ Desviación estándar

IMC = Índice de masa corporal

las primeras 24 horas de vida fueron significativas $(p<0,0001)$, en las 3 modalidades de alimentación y en ambos sexos; pero, las diferencias promedios entre los sexos no fueron significativos $(p=0,23)$. Con relación a la incidencia de hipoglucemia, según modalidades de alimentación, se observó que la mayor incidencia correspondió a los RN alimentados con LME $(16,7 \%)$, siguiéndole en orden decreciente los RN alimentados con leche materna más leche maternizada de inicio (10\%); no observándose ningún caso en los RN alimentados con leche materna más suero glucosado; sin embargo, estas diferencias no fueron significativas $p>0,05$ (Tabla $3)$. De los cinco casos de hipoglucemia en RN alimentados con LME, uno ocurrió a las 2 horas de vida y cuatro a las 4 horas (Tabla 4). En los tres casos de hipoglucemia en los RN alimentados con leche materna más leche maternizada de inicio, éstos se presentaron a las 4 horas de vida (Tabla 5).
Asimismo, se encontró que los RN alimentados con LME presentaron mayor pérdida ponderal a las 24 horas de vida, seguido por el grupo de RN alimentados con leche materna más suero glucosado, y los alimentados con leche materna más leche maternizada de inicio, respectivamente, siendo estas diferencias significativas $p=$ 0,046 (Tabla 6).

\section{DISCUSIÓN}

La OMS, en los artículos 1 y 2 del Código Internacional de Comercialización de Sucedáneos de Leche Materna, en la Resolución WHA 39,28 de la 39a Asamblea de la OMS, del 16-05-86, refiere que durante los primeros cuatro a seis meses de vida, la leche materna sola es usualmente adecuada para satisfacer las necesidades nutricionales de un RN normal $\left({ }^{21}\right)$. La Academia Americana de Pediatría, desde sus comienzos, se 
Custodio Quispe y col.

Tabla 2. Niveles de glucosa en recién nacidos según modalidad de alimentación, sexo y edad postnatal.

\begin{tabular}{|c|c|c|c|c|c|c|c|c|c|c|c|c|}
\hline \multirow{3}{*}{$\begin{array}{c}\text { Edad } \\
\text { posnatal } \\
\text { en horas }\end{array}$} & \multicolumn{4}{|c|}{$\begin{array}{l}\text { Leche materna } \\
\text { exclusiva }\end{array}$} & \multicolumn{4}{|c|}{$\begin{array}{l}\text { Leche materna más leche } \\
\text { maternizada de inicio }\end{array}$} & \multicolumn{4}{|c|}{$\begin{array}{l}\text { Leche materna más } \\
\text { suero glucosado }\end{array}$} \\
\hline & \multicolumn{2}{|c|}{ Varones } & \multicolumn{2}{|c|}{ Mujeres } & \multicolumn{2}{|c|}{ Varones } & \multicolumn{2}{|c|}{ Mujeres } & \multicolumn{2}{|c|}{ Varones } & \multicolumn{2}{|c|}{ Mujeres } \\
\hline & Promedio & $\overline{\mathrm{DE}}$ & Promedio & $\overline{\mathrm{DE}}$ & Promedio & $\overline{\mathrm{DE}}$ & Promedio & $\overline{\mathrm{DE}}$ & Promedio & $\overline{\mathrm{DE}}$ & Promedio & $\overline{\mathrm{DE}}$ \\
\hline Al nacer & 67,8 & 16,7 & 62,1 & 11,6 & 69,9 & 13,4 & 61,2 & 15,5 & 73,8 & 14,9 & 66,1 & 15,2 \\
\hline 2 & 55,9 & 9,0 & 54,9 & 6,7 & 53,5 & 8,8 & 56,3 & 11,0 & 55,3 & 9,4 & 57,7 & 9,2 \\
\hline 4 & 56,1 & 9,4 & 51,3 & 6,4 & 62,5 & 14,1 & 55,2 & 12,9 & 61,7 & 19,0 & 60,9 & 12,4 \\
\hline 6 & 59,7 & 9,9 & 61,4 & 7,1 & 64,2 & 10,6 & 61,5 & 15,2 & 67,0 & 16,1 & 65,3 & 10,6 \\
\hline 12 & 65,7 & 8,4 & 60,9 & 9,5 & 69,7 & 16,4 & 66,8 & 12,1 & 70,2 & 11,8 & 66,1 & 13,0 \\
\hline 18 & 61,9 & 11,0 & 64,4 & 7,0 & 72,6 & 16,9 & 71,9 & 11,8 & 73,1 & 9,3 & 70,2 & 9,7 \\
\hline 24 & 64,9 & 8,6 & 67,0 & 6,2 & 68,7 & 10,9 & 65,1 & 12,6 & 70,7 & 13,6 & 74,1 & 11,7 \\
\hline Total & 61,7 & 10,42 & 60,3 & 7,78 & 65,9 & 13,0 & 54,7 & 13,0 & 67,4 & 13,4 & 65,8 & 11,7 \\
\hline
\end{tabular}

DE = Desviación estándar.

$p<0,0001$ (según edad posnatal entre los grupos), $p=0,23$ (entre sexos), según ANOVA multivariable para muestras repetidas.

convirtió en defensora a ultranza de la lactancia materna, como forma óptima de alimentación para el lactante. La leche humana es netamente superior para la alimentación del niño $\left(^{7}\right)$. La Iniciativa Hospitales Amigos del Niño y de la Madre (IHANM) es una estrategia válida para la lactancia exitosa. Los RN deben recibir alimentación con LME hasta que logren saciarse, generalmente tras 10 a 15 minutos de tetada, en cada pecho, a libre demanda (8 a 12 veces, en 24 horas) ${ }^{9,22}$. Todos los RN del presente estudio han sido puestos en contacto precoz piel a piel con sus madres, a fin de iniciar y estimular la succión del pezón materno, para mejorar la producción de le-

Tabla 3. Incidencia de hipoglucemia según modalidades de alimentación.

\begin{tabular}{lcc}
\hline \multicolumn{1}{c}{$\begin{array}{c}\text { Modalidad } \\
\text { de alimentación }\end{array}$} & $\begin{array}{c}\text { Número } \\
\text { de casos }\end{array}$ & $\begin{array}{c}\text { Tasa de } \\
\text { incidencia \% }\end{array}$ \\
\hline $\begin{array}{l}\text { Leche materna exclusiva } \\
\text { Leche materna más leche } \\
\text { maternizada de inicio }\end{array}$ & 5 & 16,7 \\
$\begin{array}{l}\text { Leche materna más } \\
\text { suero glucosado }\end{array}$ & 0 & 10,0 \\
\hline
\end{tabular}

Chi cuadrado $p>0,05$ che. El contacto piel a piel o el contacto físico estrecho entre la madre y el hijo mantiene la temperatura adecuada del neonato, mejorando el apego madre-niño $\left({ }^{5,22-24}\right)$. Gray (2 000) $\left({ }^{25}\right)$, en un estudio sobre el efecto analgésico del contacto piel a piel, concluye que éste constituye una intervención potente contra el dolor sufrido por los RN sanos, tal como ocurre al pinchar el talón del pie para obtener una muestra de sangre. En un estudio posterior (2002), Gray $\left({ }^{26}\right)$ demuestra que la lactancia materna tiene un potente efecto analgésico contra el dolor producido por procedimientos invasivos en el RN. Las características maternas fueron similares en las diversas mo-

Tabla 4. Niveles de glucemia por edad posnatal en los 5 casos de hipoglucemia en recién nacidos alimentados con lecha materna exclusiva.

\begin{tabular}{cccccccc}
\hline \multirow{2}{*}{ Casos } & \multicolumn{7}{c}{ Edad posnatal } \\
\cline { 2 - 8 } & Al nacer & $2 \mathrm{~h}$ & $4 \mathrm{~h}$ & $6 \mathrm{~h}$ & $12 \mathrm{~h}$ & $18 \mathrm{~h}$ & $24 \mathrm{~h}$ \\
\hline 1 & 59 & 55 & $\mathbf{3 9}$ & 59 & 60 & 54 & 60 \\
2 & 45 & 50 & $\mathbf{3 8}$ & 58 & 56 & 54 & 59 \\
3 & 48 & $\mathbf{3 7}$ & 56 & 57 & 51 & 57 & 76 \\
4 & 53 & 40 & $\mathbf{3 9}$ & 56 & 60 & 56 & 61 \\
5 & 73 & 54 & $\mathbf{3 9}$ & 60 & 65 & 76 & 66 \\
\hline
\end{tabular}


Tabla 5. Niveles de glucemia por edad postnatal en los 3 casos de hipoglucemia en recién nacidos alimentados con lecha materna más leche maternizada de inicio.

\begin{tabular}{cccccccc}
\hline \multirow{2}{*}{ Casos } & \multicolumn{7}{c}{ Edad posnatal } \\
\cline { 2 - 8 } & Al nacer & $2 \mathrm{~h}$ & $4 \mathrm{~h}$ & $6 \mathrm{~h}$ & $12 \mathrm{~h}$ & $18 \mathrm{~h}$ & $24 \mathrm{~h}$ \\
\hline 1 & 77 & 69 & $\mathbf{3 6}$ & 60 & 51 & 61 & 61 \\
2 & 96 & 67 & $\mathbf{3 4}$ & 60 & 78 & 70 & 65 \\
3 & 64 & 42 & $\mathbf{3 5}$ & 57 & 57 & 59 & 58 \\
\hline
\end{tabular}

dalidades de alimentación, salvo la hemoglobina, cuyo promedio fue mayor en las madres de los RN que fueron alimentados con leche materna más suero glucosado $(11,7 \mathrm{~g} / \mathrm{dL})$, siguiéndoles en orden decreciente las madres de los RN alimentados con $\operatorname{LME}(11,3 \mathrm{~g} / \mathrm{dL})$ y por último las madres de los RN alimentados con leche materna más leche maternizada de inicio $(10,8$ $\mathrm{g} / \mathrm{dL})$. Estas diferencias pueden ser debido a que no todas las madres tienen el mismo nivel socioeconómico, lo que en alguna forma puede repercutir sobre su estado nutricional. Con relación a las características de los RN, observamos que las cifras promedio fueron similares en los tres grupos, salvo el perímetro cefálico, cuyo pro- medio fue mayor en RN alimentados con leche materna más leche maternizada, que fue el grupo que también presentó el mayor promedio ponderal, y de la talla. Además, se sabe que los RN presentan diversos grados de modelaje craneano, debido al trabajo de parto, y por consiguiente las diferencias en la medición del perímetro cefálico son menores a las 24 horas de vida.

Se refiere que el niño nace con un nivel de glucemia aproximado de $70 \%$ de su madre $\left({ }^{5,27-29}\right)$. En el presente estudio se encontró que la glucemia del RN fue $62,4 \%$ de la glucemia materna, lo que se explicaría porque las tomas de las muestras de sangre se realizaron dentro de la primera media hora, después de nacer y no inmediatamente después del nacimiento.

Con relación a la evolución de los niveles de glucemia en las primeras 24 horas de vida, observamos que, tanto en $\operatorname{los} \mathrm{RN}$ alimentados con LME como en los alimentados con leche materna más leche maternizada, ésta disminuye en los varones en las dos primeras horas para luego comenzar a ascender, a partir de las 4 horas de vida, mientras que en las mujeres, la

Tabla 6. Recién nacidos por pérdida ponderal a las 12 y 24 horas de vida, según modalidad de alimentación.

\begin{tabular}{|c|c|c|c|c|c|}
\hline \multirow[t]{2}{*}{ Modalidad de alimentación } & \multicolumn{5}{|c|}{ Peso en gramos } \\
\hline & Al nacer & A las $12 \mathrm{~h}$ & Pérdida ponderal \%* & A las $24 \mathrm{~h}$ & Pérdida ponderal \%* \\
\hline \multicolumn{6}{|l|}{ Leche materna exclusiva } \\
\hline Promedio & 3319,0 & 3233,3 & 2,6 & 3144,8 & 5,2 \\
\hline Desviación estándar & 267,2 & 252,3 & & 253,1 & \\
\hline \multicolumn{6}{|c|}{ Leche materna más leche maternizada de inicio } \\
\hline Promedio & 3467,3 & 3394,3 & 2,1 & 3324,7 & 4,1 \\
\hline Desviación estándar & 302,3 & 291,1 & & 278,4 & \\
\hline \multicolumn{6}{|c|}{ Leche materna más suero glucosado } \\
\hline Promedio & 3325,3 & 3227,0 & 3,0 & 3158,3 & 5,0 \\
\hline Desviación estándar & 328,9 & 311,5 & & 303,9 & \\
\hline
\end{tabular}

$n=90$ recién nacidos.

* Pérdida ponderal con relación al peso de nacimiento.

$p<0,0001$ (en el tiempo), $p=0,046$ (entre los grupos) según ANOVA multivariante, para muestras repetidas. 
disminución se prolonga hasta las 4 horas y recién empieza a ascender a las 6 horas; sin embargo, en los RN alimentados con leche materna más suero glucosado, esta tendencia al descenso tiene una duración más corta (hasta las 2 horas de vida) y se incrementa a partir de las 4 horas, tanto en varones como en mujeres. Hoseth $\left({ }^{2}\right)$ encontró que la glucosa sanguínea disminuye en la primera hora de vida en $42 \%$ y luego tiene una tendencia irregular con incrementos y descensos, hallándose los niveles más bajos a las 6 y 12 horas de vida. Srinivasan (1986) $\left({ }^{30}\right)$, hizo un estudio de 344 neonatos, sanos y adecuados para la edad gestacional, alimentados con leche maternizada (20 calorías por onza), después de 3-4 horas de vida y alimentados cada 4 horas; el 10 a $15 \%$ de los lactantes recibieron leche materna; encontró promedios de glucosa plasmática $(\mathrm{mg} / \mathrm{dL})$ de 107 al nacer, de 56 a la hora, de 60 a las 2 horas, de 70 a las 3 horas, de 68 a las 4 horas, de 65 a las 6 horas y de 67 a las 12 a 24 horas de vida, cifras similares a las halladas por nosotros en las primeras 24 horas de vida. Por otra parte, Heck (1987) ${ }^{(31}$ ) estudió 114 RN a término, 64 alimentados con leche materna y 50 con leche maternizada, hallando que el percentil 50 de los valores de la glucosa sérica en ambos grupos fue $90 \mathrm{mg} / \mathrm{dL}$, en sangre de cordón; luego, a la hora de vida, $56 \mathrm{mg} / \mathrm{dL}$, a las 2 horas $58 \mathrm{mg} / \mathrm{dL}$, a las 5 a 6 horas $56 \mathrm{mg} / \mathrm{dL}$, a las 10 a 14 horas 56 $\mathrm{mg} / \mathrm{dL}$ y a las 20 a 28 horas $60 \mathrm{mg} / \mathrm{dL}$. Hawden (1992) ${ }^{(32}$ ), en un estudio transversal de la concentración de la glucosa sanguínea, en $156 \mathrm{RN}$ a término, alimentados con leche materna y leche maternizada a libre demanda, encontró promedios de glucosa sanguínea al nacer de $77,4 \mathrm{mg} / \mathrm{dL}$, a la 1 a 12 horas, $55,8 \mathrm{mg} / \mathrm{dL}$, y a las 12 a 24 horas, $66,6 \mathrm{mg} / \mathrm{dL}$; los niños alimentados con leche materna $(46 \%$ de la muestra), a la semana de vida tenían niveles bajo de glucosa sanguínea, en comparación con los alimentados con leche maternizada, con promedios de $64,8 \mathrm{mg} / \mathrm{dL}$ y $72 \mathrm{mg} / \mathrm{dL}$, res- pectivamente, siendo esta diferencia significativa $(p<0,05)$.

La incidencia de hipoglucemia en los tres grupos fue desigual. En el grupo de RN alimentados con LME, 16,7\% (5 de 30) presentó valores de glucemia menores a 40 $\mathrm{mg} / \mathrm{dL}$, mientras que en el grupo de RN con leche materna más leche maternizada de inicio, solo $10 \%$ (3 de 30) y ninguno en los RN alimentados con leche materna más suero glucosado. Todos estos casos de hipoglucemia fueron asintomáticos (Tabla 3). Identificados los neonatos con hipoglucemia, éstos fueron inmediatamente apoyados con leche maternizada de inicio a libre demanda, con volúmenes no menores de $20 \mathrm{~mL}\left({ }^{5,33}\right)$; los controles de glucemia 30 minutos después evidenciaron su elevación a niveles normales (Tabla 4). Ninguna de las cinco madres recibieron dextrosa parenteral antes del período expulsivo y los niveles de la glucemia materna estaban dentro de límites normales. Los tres casos de hipoglucemia, de RN alimentados con leche materna más leche maternizada de inicio, recibieron inmediatamente $15 \mathrm{~mL}$ de suero glucosado y luego $15 \mathrm{~mL}$ de leche maternizada de inicio, mejorando rápidamente sus niveles de glucemia (Tabla 5). A este hecho, Volpe (2003) ${ }^{34}$ ) denomina hipoglucemia transitoria adaptativa temprana $\mathrm{y}$, por lo general, ocurre durante las primeras 6 a 12 horas después de la supresión repentina del sustrato derivado de la madre, en el momento del nacimiento. Estos RN no hacen los ajustes metabólicos de adaptación apropiados durante la transición de la vida intrauterina a la extrauterina; es de duración breve, de leve severidad y la respuesta a la administración de glucosa es rápida. Además, refiere que la incidencia de hipoglucemia neonatal varía mucho con la edad gestacional, el momento de la investigación, la cronología y el tipo de alimentación, el control de la temperatura. En un estudio de 232 lactantes de riesgo bajo, la incidencia 
de hipoglucemia fue $21 \%$. La glucosa es esencialmente fundamental para el metabolismo cerebral y la mayor fuente de glucosa para el cerebro es suministrada por la sangre; si la glucosa sanguínea disminuye marcadamente, puede conducir a una encefalopatía severa. Es por esta razón que el diagnóstico temprano de hipoglucemia es esencial para su manejo oportuno y adecuado $(5,13,29,33,35)$. Y en vista de la posibilidad que la hipoglucemia neonatal pueda requerir futuras investigaciones, por estar asociado a posterior litigación debido a problemas médico-legales, es esencial la documentación sucinta del caso $\left({ }^{36}\right)$. García (1996), en un estudio transversal sobre «Niveles de glucemia en madres y recién nacidos» $\left({ }^{27}\right)$, de 100 neonatos a término sanos, encontró que $12 \%$ de los RN presentó niveles de glucemia menores a $40 \mathrm{mg} / \mathrm{dL}$; uno se debió a isoinmunización al grupo sanguíneo, otro a policitemia y en los 10 restantes no se encontró la causa; todos ellos fueron asintomáticos; las muestras sanguíneas fueron tomadas al momento del nacimiento, después de estar estabilizado el neonato. Diwakar $\left(^{1}\right)$, en un estudio ya mencionado, encontró valores de glucosa plasmática menores a $40 \mathrm{mg} / \mathrm{dL}$, en $16,5 \%$, a las 3 horas de vida, y de $10 \%$ a las 6,24 y 72 horas, respectivamente; pero, los promedios de glucosa plasmática no variaron significativamente entre las 3 y 72 horas de vida. Por su parte, Srinivasan $\left({ }^{30}\right)$, a la hora de vida encontró un $12 \% \quad(6 / 52)$ de hipoglucemia neonatal, a las 2 horas $2 \%$ $(1 / 52)$ y ninguno a partir de las 3 horas. Heck ${ }^{31}$ ) encontró valores de glucosa sérica menor a $40 \mathrm{mg} / \mathrm{dL}$ en $16 \%$ de las muestras estudiadas. Mc Gowan $\left({ }^{29}\right)$ refiere una incidencia de hipoglucemia neonatal de 1 a 5 por mil nacidos vivos, pero es alto en RN con factores de riesgo para hipoglucemia. Sexson $\left({ }^{4}\right)$ encontró $21 \%$ de hipoglucemia en todos los lactantes.

La dextrosa al 5\% se metaboliza rápidamente; la leche contiene más energía por mililitro que la dextrosa al 5\% y su alto contenido de grasa provee de sustratos para la cetogénesis $\left({ }^{5}\right)$. El uso de leche maternizada provee de carbohidratos (bajo la forma de lactosa), de proteínas y grasas que son metabolizadas lentamente, lo que permite proveer sustratos en forma prolongada. Se ha estimado que la concentración de la glucosa sanguínea incrementa a $30 \mathrm{mg} / \mathrm{dL}$ dentro de la primera hora después de una alimentación de 30 a $60 \mathrm{~mL}$ de leche maternizada $\left({ }^{29}\right)$. Si la concentración de glucosa sanguínea posprandial no ha mejorado, se considera que el tratamiento oral ha fallado y el niño debe recibir inmediatamente tratamiento endovenoso de glucosa $\left({ }^{29,33}\right)$.

La pérdida fisiológica del peso en las primeras 12 y 24 horas de vida fue mayor en los RN alimentados con $\operatorname{LME}(5,2 \%)$, siguiéndole en orden decreciente los alimentados con leche materna más suero glucosado $(5,0 \%)$ y leche materna más leche maternizada de inicio $(4,1 \%)$. Estos hallazgos son similares a los encontrados por Cairo $\left({ }^{37}\right)$, quien refiere que la pérdida ponderal durante el primer día de hospitalización fue menor en los RN que recibieron apoyo con leche maternizada, comparado con el grupo que recibió lactancia materna exclusiva. Heck $\left({ }^{31}\right)$ encontró una mayor pérdida ponderal en los RN alimentados con LME que con leche maternizada $(5,98 \%$ vs. $2,8 \%)$. Dollberg $\left({ }^{38}\right)$ halló un mayor volumen lácteo ingerido en el primer día de vida en los RN alimentados con leche maternizada, que los alimentados con LME. De manera similar, son los hallazgos de Casey $\left({ }^{39}\right)$ y Evans $\left({ }^{40}\right)$, en sus estudios sobre la ingesta de leche materna. También, se puede utilizar el suero glucosado o dextrosa al 5\%, ya que aumentan rápidamente los niveles de glucemia, por tener un índice glicémico de 100; y, al administrar leche maternizada, que tiene lactosa, proteínas y grasas, las que se metabolizan lentamente, permiten proveer una fuente más sostenida de sustratos para la gluconeogénesis, ya que 
tiene un índice glucémico de 30. Respecto al apetito, los alimentos con un bajo índice glucémico tienden a producir mayor saciedad, que los alimentos con un alto índice glucémico $\left({ }^{29,41,42}\right)$. Chen $(1998)\left({ }^{43}\right)$ refiere que la primiparidad, el parto prolongado, el estrés de la madre y el feto durante los periodos de dilatación y expulsivo del parto, y la glucemia elevada del cordón son factores de riesgo para la lactogénesis retardada y recomienda apoyar a estos niños con leche maternizada y su seguimiento en el puerperio inmediato, para educar a la madre y mejorar el éxito de la lactancia materna. El estrés (ansiedad, depresión y disconfórt), la enfermedad materna y las complicaciones del parto pueden afectar la producción láctea. El clínico, para proteger la lactancia en periodos de estrés, debe intervenir, facilitando la lactancia y aliviando el estrés $\left({ }^{5,23,33,44}\right)$. De acuerdo a lo observado, tanto la alimentación con LME como con leche materna más leche maternizada de inicio, no es garantía suficiente para evitar la hipoglucemia neonatal, especialmente en las primeras 4 horas de vida. Esto se debería posiblemente a que, en las primeras horas de vida, la producción adecuada de leche materna no está aún desarrollada $\left({ }^{29,33,34}\right)$ y, por consiguiente, consideramos necesaria la adición de suero glucosado a libre demanda antes de las 4 horas de vida; después de dicho período, la lactancia materna exclusiva sería suficiente para mantener la normoglucemia neonatal.

Podemos concluir que: 1) Los RN alimentados con LME tienen una tendencia a la disminución de los niveles de glucemia de duración más prolongada y un incremento más tardío que los alimentados con leche materna más leche maternizada de inicio y leche materna más suero glucosado. 2) El promedio de la glucemia neonatal al nacer fue $66,8 \mathrm{mg} / \mathrm{dL}$ y el promedio de la glucemia materna fue $109,5 \mathrm{mg} / \mathrm{dL}$, con una razón de la glucemia neonatal y glucemia materna de $62,4 \%$. 3) La incidencia de hipoglucemia asintomática fue mayor en los
RN alimentados con LME (16,7\%), siguiéndole en orden decreciente los alimentados con leche materna más leche maternizada de inicio (10\%), no hallándose ningún caso en los alimentados con leche materna más suero glucosado. 4) Los $\mathrm{RN}$ alimentados con LME tuvieron una mayor pérdida ponderal que los RN alimentados con leche materna más suero glucosado y leche materna más leche maternizada de inicio.

\section{REFERENCIAS BIBLIOGRÁFICAS}

1. Diwakar KK, Sasidhar MV. Plasma glucose levels in term infants who are appropriate size for gestation and exclusively breast fed. Arch Dis Child Fetal Neonatal. 2002;87:F46-8.

2. Hoseth E, Joergensen A, Ebbesen F, Moeller M. Blood glucose levels in a population of healthy, breast fed, term infants of appropriate size for gestational age. Arch Dis Child Fetal Neonatal. 2000;83:F117-9.

3. Eidelman AI. Hypoglycemia and the breastfed neonate. Pediatric Clinics of North America. 2001;48(2):377-87.

4. Sexson WR. Incidence of neonatal hypoglycemia: A matter of definition. The Journal of Pediatrics. 1984;105(1):14950.

5. Williams AF. Hipoglucemia del recién nacido. Ginebra: Organización Mundial de la Salud; 1997.

6. Cornblath M, Ichord R. Hypoglycemia in the Neonate. Seminars in Perinatology. 2000;24(2):136-49.

7. American Academy of Pediatrics. Lactancia materna y utilización de la leche humana. Pediatrics (ed esp.). 1997;44(6):442-5.

8. McKenna JJ, Mosko SS, Richard CA. Compartir la cama favorece la lactancia materna. Pediatrics (ed. esp.). 1997;44(2):129-34.

9. Cadwell K. Formas de alcanzar los objetivos de «Salud para todos en el año 2000» respecto al amamantamiento. Clínicas de Perinatología. 1999;2:529-40.

10. Krause. Nutrición y Dietoterapia. $8^{\text {va }}$ edición. México: Ed. Interamericana McGraw-Hill; 1995.

11. Allué IP. Fisiología de la digestión y absorción de los hidratos de carbono. Alim Nutri. Salud. 1998;5(3):74-9.

12. Ogatta ES. Homeostasis de los hidratos de carbono. En: Avery G, Fletcher M, MacDonald M. Neonatología. Fisiopatología y Manejo del Recién Nacido. 5ta edición. Buenos Aires: Ed. Médica Panamericana; 2001. p. 701-17.

13. Cornblath M, Ichord R. Hypoglycemia in the neonate. Seminars in Perinatology. 2000;24(2):136-49.

14. Mann J. Carbohydrates. En: Bowman BA, Rusell RM. Present Knowledge in Nutrition. $8^{\text {th }}$ edition. Washington, DC,: ILSI Press; 2001. p. 59-71.

15. Nordlie RC, Foster JD. Regulation of glucose production by the liver. Annu Rev Nutr. 1999;19:379-406. 
16. Chirinos R, Comparación de sistemas de medición de la glucosa sanguínea en recién nacidos del Hospital Rebagliatti. Tesis Magíster. Universidad Peruana Cayetano Heredia. Lima-Perú, febrero 2002.

17. Instituto Materno Perinatal. Manual de Organización y Procedimientos en Neonatología. Perú. Lima: MINSA; 1998.

18. Torrent RF. Problemas de adaptación metabólica del recién nacido. En: Pujol AN. Manual de Neonatología. Madrid: Mosby/Doyma Libros S.A.; 1996. p. 115-20.

19. Pérez A, Rodríguez N, Fabián J, Ramírez GA. Tamaño de la muestra, versión 1.1. Programa estadístico (CD). En: Ruiz A, Morillo LE. Epidemiología Clínica. Investigación clínica aplicada. Bogotá: Editorial Médica Panamericana; 2004.

20. Council for International Organizations of Medical Sciences (CIOMS). Normas éticas internacionales para las investigaciones biomédicas con sujetos humanos. Publicación científica $\mathrm{N}^{\circ}$ 563. Washington: OMS-OPS; 1966.

21. Ministerio de Salud. Lactancia Materna. Texto para el uso de capacitadores en la Iniciativa «Hospital Amigo de la Madre y el Niño». Lima: MINSA; 1993.

22. Philipp BL, Merewood A, Miller LW, Chawla N, MurphySmith MM, Gomes JS, et al. Baby-friendly hospital initiative improves breastfeeding initiation rates in a US hospital setting. Pediatrics. 2001;108(3):677-81.

23. Lawrence R, Lawrence RM. Approach to breast-feeding. En: Walker WA. Nutrition In Pediatrics. $3^{\text {rd }}$ edition. Canada: BC Decker Inc.; 2003. p. 562-79.

24. Fransson AL, Karlsson H, and Nilsson K. Temperature variation in newbon babies: importance of physical contact with the mother. Arch Dis Child Fetal Neonatal. 2005;90:F500-4.

25. Gray LW, Blass E. Skin-to-skin contact is analgesic in healthy newborns. Pediatrics. 2000;105(1):14-20.

26. Gray L, Miller LW, Philipp BL, Blass EM. Breastfeeding is analgesisc in healthy newborns. Pediatrics. 2002;109:590-3.

27. García RE, Rubio JL. Niveles de glicemia en madres y recién nacidos. Hospital Maternidad Rafael Calvo. Departamento de Pediatría. Facultad de Medicina. Universidad de Cartagena. Temas de Pediatría $N^{\circ}$ 141. Santa Fé de Bogotá: Nestlé; 1992. p. 1-13.

28. Cornblath M, Hawdon JM, Williams AF, Aynsley-Green A, Ward-Platt MP, Schwartz R, et al. Controversies regarding definition of neonatal hypoglycemia: suggested operational thresholds. Pediatrics. 2000;105(5):1141-5.

29. McGowan JE. Neonatal hypoglycemia. AAP Journals 1999;20:6e-15e.

30. Srinivasan G, Pildes RS, Cattamanchi G, Voora S, Lilien LD. Plasma glucose values in normal neonates: A new look. J Pediatr. 1986;109(1):114-7.

31. Heck LJ, Erenberg A. Serum glucose levels in term neonates during the first 48 hours of life. J Pediatr. 1987;110(1):11922 .
32. Hawdon JM, Ward MP, Aynsley-Green A. Patterns of metabolic adaptation for preterm and term infants in the first neoatal week. Arch Dis Child. 1992;67:357-65.

33. Thureen PJ, Deacon J, O'Neill P, Hernandez JA, editors. Assessment and care of the well newborn. $2^{\text {nd }}$ edition. USA: Elsevier Saunders, 2005.

34. Volpe, JJ. Hipoglucemia y lesión cerebral. Neurología del recién nacido. 4ta edición. México: Mc Graw Hill Interamericana; 2003. p. 531-56.

35. Traill Z, Squier M, Anslow P. Brain imaging in neonatal hypoglycaemia. Arch Dis Fetal Neonatal. 1998;79:F145-7.

36. Williams AF. Neonatal hypoglycaemia: clinical and legal aspects. Seminars in Fetal and Neonatal Medicine. 2005;10:363-8.

37. Cairo J. Lactancia materna exclusiva hasta los seis meses y factores asociados en niños nacidos sanos. An Fac Med. 2006;61(3):193-200.

38. Dollberg S, Lahay S, Mimouni FB. A Comparison of intakes of breast-fed and bottle-fed infants during the first two days of life. J Am College of Nutrition. 2001;20(3):209-11.

39. Casey CE, Neifert MR, Seacat JM, Neville MC. Nutrient intake by breast-fed infants during the first five days after birth. AJDC. 1986;140:933-66.

40. Evans KC, Evans RG, Royal R, Esterman AJ, James SL. Effects of cesarean section on breast milk transfer to the normal term newborn over the first week of life. Arch Dis Child Fetal Neonatal. 2003;88:F380-2.

41. Foster-Powell K, Miller JB. International tables of glycemic index. Am J Clin Nutr. 1995;2:871s-93s.

42. Foster-Powell K, Holt S, Brand-Miller J. International table of glycemic index and glycemic load values. Arm J Clin. Nutr. 2002;76:5-56.

43. Chen DC, Nommsen-Rivers L, Dewey KG, Lonnerdal B. Stress during labor and delivery and early lactation performance. Am J Clin Nutr. 1998;68:335-44.

44. Lau Ch. Effects of stress on lactaction. Breasrfeeding. 2001, Part 1. Pediatric Clinics of North America. 2001;48(1):22134.

Manuscrito recibido el 06 de agosto de 2007 y aceptado para publicación el 18 de setiembre de 2007.

Correspondencia:

Custodio Olsen Quispe Condori

Calle Manco II $N^{o}$ 388. Urb. Maranga, San Miguel.

Lima 32, Perú

Correo-e: custodioolsen@hotmail.com 